\title{
FC Portugal 2001 Team Description: Flexible Teamwork and Configurable Strategy ${ }^{\star}$
}

\author{
Nuno Lau ${ }^{1}$ and Luis Paulo Reis ${ }^{2}$ \\ 1 IEETA - Electronics and Telecomm. Eng. Institute - Aveiro University, Portugal \\ lau@det.ua.pt, http://www.ieeta.pt/ \\ ${ }^{2}$ LIACC - Artificial Intelligence and Comp. Sci. Lab. - Porto University, Portugal \\ lpreis@fe.up.pt, http://www.ncc.up.pt/liacc/
}

\begin{abstract}
FC Portugal is a cooperation project between the Universities of Aveiro and Porto in Portugal. FC Portugal 2001 is our second step towards the creation of a flexible RoboSoccer team, with tactical changing abilities, that may be coached at any level, before and during the games, by human or automatic coaches. Although having the best goal average in the competition (scoring 150 goals in 13 games), the team was not able to score against the good defenses of Tsinghuaeolus and Brainstomers and finished third in RoboCup 2001.
\end{abstract}

\section{Introduction}

FC Portugal 2001 is the result of the evolution of our previous team, FC Portugal 2000, which was RoboCup 2000 champion. For a description of FC Portugal 2000 please read [1,2]. The team achieved third place in RoboCup 2000, scoring a total of 150 goals and conceding only 5 goals. FC Portugal was stopped in RoboCup 2001 by the amazingly powerful defenses of Tsinghuaeolus and Brainstormers and although dominating spatially the games against these two teams, was not able to score against neither of them.

This paper summarizes FC Portugal 2001 main innovations and describes the team' results in RoboCup 2001. Section 2 describes our flexible team strategy and the team ability to change its tactic with and without the automatic coach intervention. Section 3 is concerned with our individual decision mechanisms and to some innovations in our low-level skills. The paper ends with the results achieved in RoboCup 2001 and its discussion.

\section{Team Strategy}

One of the main developments of FC Portugal 2001 team is the improvement of its ability to be coached both before and during the game. Before the game,

\footnotetext{
* We would like to thank the financial support from IEETA/University of Aveiro, LIACC and FEUP/University of Porto and ICEP.
} 
the strategy is defined through configuration files and during the game the tactics used may be changed by the automatic coach or even without the coach intervention by a set of rules known by all players.

The definition of strategic behaviors has been enhanced with new parameters that effectively control player movements. Active behaviors have also been extended to support different playing styles. Some of the concepts of Coach Unilang [3]; like game pace, team aggressiveness, team mentality, etc have been included in order to turn the team adaptable to the opponents strategy.

For each game a human coach specifies the strategy to be taken by the team. This strategy consists of several tactics that should be activated according to the opponent team' behavior and statistical information gathered during the game. Each tactic consists of several global parameters that control the team behavior and of several formations that are active in different situations (like defense, attack, etc). For each formation the human coach can specify the strategic and active behavior of each player. During the game players follow the coach advice to change tactics, formations and player types. Players may also decide tactical changes by themselves, based on statistical information sent by the coach and teammate and opponent modeling techniques.

FC Portugal 2001 extended FC Portugal 2000 flexible tactical approach and now, the team configuration files are far more flexible, enabling to change completely the team behavior for a given game. The information contained in the team main configuration file (strategy.conf) is summarized in Fig. 1 The first section is concerned with Player Type definition at three levels: strategic, ball possession and ball recovery. The second section enables us to give players information about the opponent team (at several levels) and about the type of game and tournament the team is involved. Other important information concerns the type of coach and its powers and the team default strategy for the game (used unless the coach states the opposite). This default strategy includes the tactic to be used depending on the game result, time and other game parameters. Please see [3] for a better explanation of FC Portugal tactic definition.
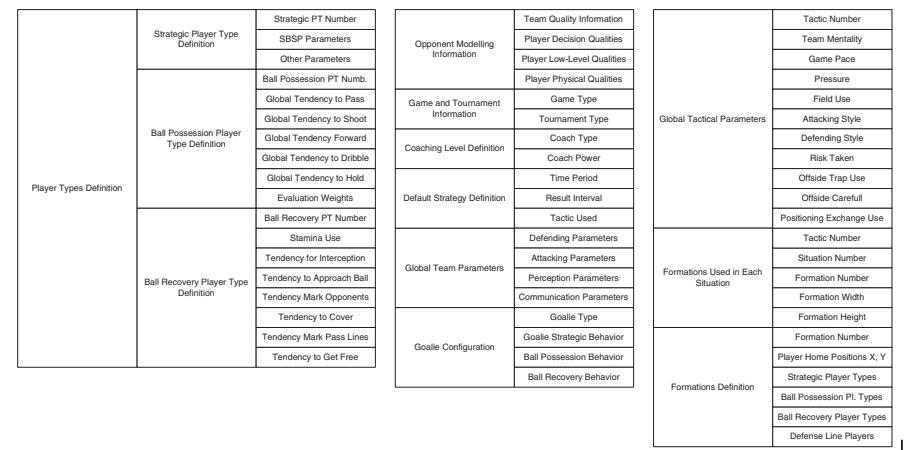

Fig. 1. FC Portugal 2001 Strategy Definition 


\section{Individual Decision and Basic Skills}

Our team considers 3 different modules in the player's behavior decision mechanism. In strategic situations the player moves to a position that maximizes his utility for the team using the SBSP module 112, ball possession module is activated when the player can kick the ball, ball recovery module is responsible for behavior selection in active defending situations.

The ball possession module considers the following actions: shoot, pass, forward, dribble and hold. Each of these actions has many options in a given situation: where to shoot?, whom to pass? etc. Our decision mechanism is based on the evaluation of each option by the composition of several high level metrics. For example, passes are evaluated through 10 metrics: positional value, receiver catching probability, out of bounds probability, opponent interception possibilities, initial congestion, final congestion, pass distance, expected pass reception, confidence in receiver position and receiver shooting position. These metrics are evaluated for each of the passing options, and the best pass is selected. Similar reasoning is applied to the other ball possession behaviors and comparison of best utilities determines which is the final player decision.

The ball recovery module selects one of the following behaviors: ball interception; ball passive interception; approach ball position; cover goal; cover center or mark pass line. The selection is based on the current situation. Each player calculates a positioning matrix that includes ball position and velocity, offside lines, and all players positions, velocities, distances to ball, number of cycles to intercept the ball. The decision is based on the analysis of the effect of each of the items of the positioning matrix. Coordination is achieved through prediction of teammates' decisions based on mutual knowledge of their decision rules.

FC Portugal 2001 also improved some of the basic skills of our previous team. The optimization kick was enhanced to optimize the difficulty of opponent interception while kicking and in the first cycles after the ball is released. Dribbling ability was changed in order to turn it faster and safer from opponent steals. A new algorithm for ball interception has been developed. In this algorithm the interception point is chosen taking into account how risky is the interception, i.e., players choose risky interception points in disputed balls and safer interceptions when they are clearly the balls owners.

\section{Results and Discussion}

FC Portugal 2001 dominated round robin qualification groups of RoboCup 2001, scoring 138 goals without conceding a single goal (Tab. 11). The team won the first game of double elimination by 8-0 against YowAI 2001 (RoboCup Japan 2001 Champion). The next game against Uva TriLearn showed FC Portugal ability to perform dramatic tactical changes. On the second half of the game, around 4000 cycles, FC Portugal was losing 1-0 and even though the team seemed to have the power to reverse the game, it was with lots of difficulties to pressure the opponent and score a goal. At this time the team changed to an aggressive tactic 
Table 1. Scores of FCPortugal 2001 in RoboCup 2001

\begin{tabular}{lc}
\hline Group E- Round Robin 1 & Score \\
\hline TUT-Groove (Japan) & $9-0$ \\
RoboLog2k1 (Germany) & $8-0$ \\
RMIT-Goannas (Australia) & $32-0$ \\
11Monkeys3 (Japan) & $29-0$ \\
\hline Group C - Round Robin 2 & \\
\hline ATTUnited01 (USA) & $22-0$ \\
FC Tripletta (Japan) & $4-0$ \\
AT Humboldt. (Germany) & $13-0$ \\
UTUtd (Iran) & $16-0$ \\
Helli-Respina 2001 (Iran) & $5-0$ \\
\hline Double elimination & \\
\hline YowAI 2001 (Japan) & $8-0$ \\
Uva Trilearn 2001 (Netherlands) & $4-1$ \\
Tsinghuaeolus (China) & $0-3$ \\
Brainstormers 2001 (Germany) & $0-1$ \\
\hline Total Score & $150-5$ \\
\hline
\end{tabular}

\begin{tabular}{llc}
\hline Team & Games & Total \\
\hline 1 Tsinghuaeolus (China) & 12 & $90-1$ \\
2 Brainstormers 2001 (Germany) & 16 & $79-5$ \\
3 FC Portugal 2001 & 13 & $150-5$ \\
4 Uva Trilearn 2001 (Netherlands) & 13 & $46-14$ \\
5 FC Portugal 2000 & 12 & $121-13$ \\
6 Wright Eagle (Japan) & 12 & $109-6$ \\
7 YowAI 2001 (Japan) & 11 & $64-15$ \\
8 FC Tripletta (Japan) & 12 & $30-16$ \\
\hline
\end{tabular}

using a 443 formation (without a goalie) for attacking. Afterwards FC Portugal dominated the game clearly and the final result (4-1), showed the importance of having tactical changing abilities. RoboBase 4 statistics show that the ball stayed mostly in the FC Portugal's attack (42\%) and midfield (42\%) with little time in defense (16\%). In the next game, against Tsinghuaeolus, FC Portugal 2001 was, again, loosing by 1-0 near the end of the game. The team had already tried several different tactics, with clear spatial dominion (final results are of $36 \%$ in attack, $55 \%$ in midfield and $9 \%$ in defense), the risky tactic the team chose at the end allowed Tsinghuaeolus to score 2 more goals. Our last game was against BrainStormers, perhaps the best defense in this year championship. Again, our team dominated spatially (46\% attack $33 \%$ midfield $21 \%$ defense) but the German team scored a goal that gave them the victory. It should be noted that the 4 top teams of RoboCup 2001 showed different approaches to the game and all of them proved to be really effective. This shows that research in the simulation league is far from coming to a dead end and a lot of different techniques are still to be evaluated.

\section{References}

1. Luis Paulo Reis and Nuno Lau, FC Portugal Team Description: RoboCup 2000 Simulation League Champion, in P.Stone et all editors, RoboCup-2000: Robot Soccer World Cup IV, Springer LNAI 2019, pp.29-40, 2001

2. Luis Paulo Reis, Nuno Lau, Eugenio Oliveira. Situation Based Strategic Positioning for Coordinating a Simulated RoboSoccer Team, in M.Hannebauer et al eds, Balancing React. and Social Delib. in MAS, Springer LNAI 2103, pp.175-197, 2001

3. Luis Paulo Reis and Nuno Lau. COACH UNILANG - A Standard Language for Coaching a (Robo)Soccer Team, in A.Birk et all editors, RoboCup-2001: Robot Soccer World Cup V, Springer, LNAI, 2002 (to appear)

4. John Sear. RoboBase - Extensively Repository and LogPlayer Supporting Immediate Remote Access, in A.Birk et all editors, RoboCup-2001: Robot Soccer World Cup V, Springer, LNAI, 2002 (to appear) 